\title{
Significance of interstitial tumor-associated macrophages in the progression of lung adenocarcinoma
}

\author{
BING-SHENG SUN ${ }^{1 *}$, BAO-XIANG PEI ${ }^{2}$, KANG ZHANG $^{2 *}$, LU-CHANG ZHANG $^{2}$, \\ GUANG-JING ZHANG ${ }^{2}$, JI-KUAN LIU ${ }^{2}$, HONG-WEI CUI ${ }^{2}$, FEN PAN $^{2}$ and ZHEN-FA ZHANG ${ }^{1}$ \\ ${ }^{1}$ Department of Lung Cancer, Tianjin Medical University Cancer Institute and Hospital, Tianjin Lung Cancer Center, \\ National Clinical Research Center of Cancer, Key Laboratory of Cancer Prevention and Therapy, Tianjin 300060; \\ ${ }^{2}$ Department of Thoracic Surgery, Jining No. 1 People's Hospital, Jining, Shandong 272011, P.R. China
}

Received July 7, 2015; Accepted September 22, 2016

DOI: $10.3892 / 01.2016 .5270$

\begin{abstract}
Stepwise progression from adenocarcinoma in situ (AIS) and minimally invasive adenocarcinoma (MIA) to lepidic predominant adenocarcinoma (LPA) was proposed by various scholars. Interstitial tumor-associated macrophages (TAMs) and various potential chemokines involved in the progression from AIS/MIA to LPA were hypothesized. In the present study, immunohistochemistry or immunofluorescent double staining was used to detect the expression of the TAMs marker cluster of differentiation (CD) 68, tumor-derived colony-stimulating factor (CSF)-1, interleukin (IL)-6, matrix metalloproteinase (MMP)-2, E-cadherin and Snail in lung adenocarcinoma specimens, including AIS/MIA, LPA and other types. It was observed that infiltrating TAMs were negatively associated with the prognosis of patients, and that the infiltration degree of interstitial TAMs was higher in LPA than that in AIS/MIA. In addition, E-cadherin, Snail and MMP-2 expression were significantly correlated with the infiltration degree of TAMs. Survival analysis revealed that co-expression of CD68, CSF-1 and IL-6 was an independent prognostic factor. Stratified analysis demonstrated that, in AIS/MIA patients, there was a statistically significant difference between the number of TAMs (TAMs $\leq 25$ and TAMs $>25$ ) in the CD68+CSF-1+IL-6+ group compared with other groups (including CD68+CSF-1-IL-6-, CD68+CSF-1+IL-6-, CD68+CSF-1-IL-6+ and CD68- groups). By contrast, in patients with TAMs $>25$ and in patients with
\end{abstract}

Correspondence to: Professor Zhen-Fa Zhang, Department of Lung Cancer, Tianjin Medical University Cancer Institute and Hospital, Tianjin Lung Cancer Center, National Clinical Research Center of Cancer, Key Laboratory of Cancer Prevention and Therapy, Huanhu West Road, He-Xi, Tianjin 300060, P.R. China E-mail: zhangzf1979@163.com

*Contributed equally

Key words: epithelial-mesenchymal transition, tumor-associated macrophages, lung adenocarcinoma, progression, colony-stimulating factor-1, interleukin-6 positive CD68, CSF-1 and IL-6 expression, the survival rates were not significantly different between AIS/MIA and LPA. These results suggested that co-expression of TAMs marker CD68, CSF-1 and IL-6 may be a valuable independent prognostic predictor in lung adenocarcinoma. TAMs may facilitate AIS/MIA progression to LPA, which may be closely associated with the induction of the epithelial-mesenchymal transition.

\section{Introduction}

Lung adenocarcinoma is the most frequent histological type of lung cancer, and the rates of adenocarcinoma are increasing in the majority of countries (1). An important step in tumor progression is the acquisition of invasive properties by tumor cells. Within lung adenocarcinoma, certain scholars suggested that invasiveness may represent the developmental process of tumor from adenocarcinoma in situ (AIS) and minimally invasive adenocarcinoma (MIA) to lepidic predominant adenocarcinoma (LPA) (2). The theoretic transition of AIS/MIA to LPA as a model was thus proposed in our study.

Recent studies have shown that the tumor microenvironment and the neoplastic cells act in concert to promote the growth and progression of the tumor mass $(3,4)$. In the stroma of several tumor types, a critical role has been reported for tumor-associated macrophages (TAMs), which exist in the tumor microenvironment (5). Although there is a growing body of preclinical and clinical evidence associating TAMs with poor prognosis of cancer patients (5), their roles in the progression of lung adenocarcinoma remain mostly unknown. A characterized progression that epithelial-derived tumor cells undergo is termed the epithelial-mesenchymal transition (EMT), which involves loss of polarity and adhesion, increased mobility and invasiveness, and acquisition of an invasive mesenchymal phenotype (6). However, the role of TAMs and their association with EMT in the progression from AIS/MIA to LPA are still unclear.

The present study investigated the clinical differences between AIS/MIA and LPA. We hypothesized that the tumor microenvironment serves a role in such differences. The present study intended to directly detect the expression of the TAMs marker cluster of differentiation (CD) 68 and a number of potential chemokines that are associated with invasion and 
progression of cancer cells, and to explore their prognostic value in lung adenocarcinoma, as well as to further study the association between TAMs and EMT. It was of great significance to further clarify the biological function of TAMs in the development of lung adenocarcinoma, and thereby open up a new avenue for the comprehensive treatment of cancer.

\section{Materials and methods}

Tissue samples. A cohort of 285 consecutive patients who received complete pulmonary resection and systematic lymph node dissection for stage I-IIIA lung adenocarcinoma (37 cases of AIS/MIA, 127 cases of LPA and 121 cases of other cancer types) at Tianjin Medical University Cancer Institute and Hospital (Tianjin, China) from September 2004 to September 2008 were included in the present retrospective study. Inclusion criteria were as follows: i) No reception of neoadjuvant therapy; ii) no presence of metastatic diseases pre-operatively; iii) complete clinicopathological and follow-up data available; and iv) survival of $>1$ month after surgery. The histology of all cases was re-assessed by two independent pathologists (Professor B.S. Sun and Dr Z.F. Zhang) according to the latest pathological classification (2). Tumor staging was based on the International Association for the Study of Lung Cancer tumornode-metastasis (TNM) 7th classification system (7). All patients were followed up until September 1, 2013. The mean follow-up time was 42 months (ranging from 2 to 100 months). Patients who were still alive after the last follow-up and who were lost to follow-up were censored in the present study. The research ethics committee of Tianjin Cancer Institute and Hospital (Tianjin, China) provided ethical approval for the study of human subjects, and all patients provided written informed consent.

Tissue microarray and immunohistochemistry or immunofluorescent double staining. For tissue microarray construction, two experienced pathologists (Professor B.S. Sun and Dr Z.F. Zhang) reviewed the hematoxylin and eosin-stained sections from each paraffin-embedded, formalin-fixed block. The most representative areas of the tumor regions were carefully selected and sampled for tissue microarray collector blocks.

Immunohistochemistry was performed with mouse antihuman CD68 monoclonal antibody at 1:5 dilution (clone KP1; Abcam, Cambridge, UK), rabbit anti-human colony-stimulating factor (CSF)-1 polyclonal antibody at 1:100 dilution (BA0750; Wuhan Boster Biological Technology, Ltd., Wuhan, China), anti-interleukin (IL)-6 at 1:100 dilution (BS0781R; BIOSS, Beijing, China), anti-matrix metalloproteinase (MMP)-2 at 1:400 dilution (ab37150; Abcam), anti-E-cadherin at 1:200 dilution (sc-7,870; Santa Cruz Biotechnology, Inc., Dallas, TX, USA) and anti-Snail at 1:25 dilution (AP2054a; Abgent, Inc., San Diego, CA, USA). Immunofluorescent double staining was used to detect CD68 (1:10 dilution; clone KP1; Abcam) and E-cadherin (1:300 dilution; sc-7870, Santa Cruz Biotechnology, Inc.). After deparaffinization, rehydration and heat-induced antigen retrieval, tissues were immersed in methanol containing 3\% hydrogen peroxide for $20 \mathrm{~min}$. Then, the sections were incubated with the above primary antibodies overnight at $4^{\circ} \mathrm{C}$. Following $30 \mathrm{~min}$ of incubation with the corresponding horseradish peroxidase-labeled secondary antibody (1:200 dilution; PV-6000; Beijing Zhongshan Golden Bridge Biotechnology Co. Ltd., Beijing, China) or fluorochrome-conjugated secondary antibody [Alexa Fluor 594-conjugated anti-rabbit immunoglobulin G (IgG) (1:400 dilution; ZF-0516; Zhongshan Golden Bridge Biotechnology Co. Ltd.) or Alexa Fluor 488-conjugated anti-mouse IgG (1:400 dilution; ZF-0512; Zhongshan Golden Bridge Biotechnology Co. Ltd.) at room temperature, the immunohistochemical sections were developed in a 3,3'-diaminobenzidine solution under microscopic observation and counterstained with hematoxylin, and the immunofluorescent sections were then stained with DAPI to visualize the nuclei. Negative control slides with the primary antibodies omitted were included in all assays.

Two pathologists (Professor B.S. Sun and Dr Z.F. Zhang) independently evaluated the staining of all anonymized samples. Five different areas at x400 magnification from each sample were systematically evaluated. The mean value of five scores was considered representative of one tumor. For CSF-1 and IL-6 staining in tumor cells, the sum of staining intensity ( $0=$ negative; $1=$ weak; $2=$ intermediate; and $3=$ strong) and percentage of positive tumor cells $(0=$ none or $<5 ; 1=5-25$; $2=25-50$; and $3>50 \%$ ) was calculated (8). The same staining extent evaluation was used to assess MMP-2, E-cadherin and Snail immunoreactivity. Scores between 0 and 2 were regarded as negative, while and scores between 3 and 6 were considered as positive. The number of CD68+ TAMs was analyzed as previously described (9). Briefly, the areas counted corresponded to the tumor stroma. The alveolar spaces in the tumor parts with lepidic pattern were deducted. According to the median number of stromal TAMs per high-power field (hpf) at x400 magnification, tissue samples were divided into three grades: $(-)=$ No infiltration; $(+)=$ infiltration level below the median; and $(++)=$ infiltration level above the median.

Statistical analysis. All statistical analyses were carried out using SPSS version 16.0 statistical software (SPSS, Inc., Chicago, IL, USA). The median extent of infiltration in tumor stroma was used as the cut-off point for assigning tumor samples. The association between ranked data and clinical parameters was analyzed by $\chi^{2}$ test. Overall survival (OS) was defined as the interval between surgery and mortality or last observation. Disease-free survival (DFS) was measured from the date of resection until detection of recurrent tumor or last follow-up assessment. OS and DFS were analyzed using the Kaplan-Meier method and the log-rank test, and multivariate analysis was tested with the Cox proportional hazard model. $\mathrm{P}<0.05$ was considered to indicate a statistically significant difference.

\section{Results}

Patient characteristics. For the purposes of the study, patients were excluded due to meet the aforementioned criteria: Patients received neoadjuvant therapy $(n=3)$ or succumbed within 1 month after surgery $(n=2)$, thus leaving 285 cases for analysis (Table I). The median age of the patients at the time of surgery was 60 years (range, 36-79 years). Of all patients, $47.0 \%$ were men, $47.0 \%$ were former or current smokers, and $44.2 \%$ had stage I disease. Patients were classified into three categories 


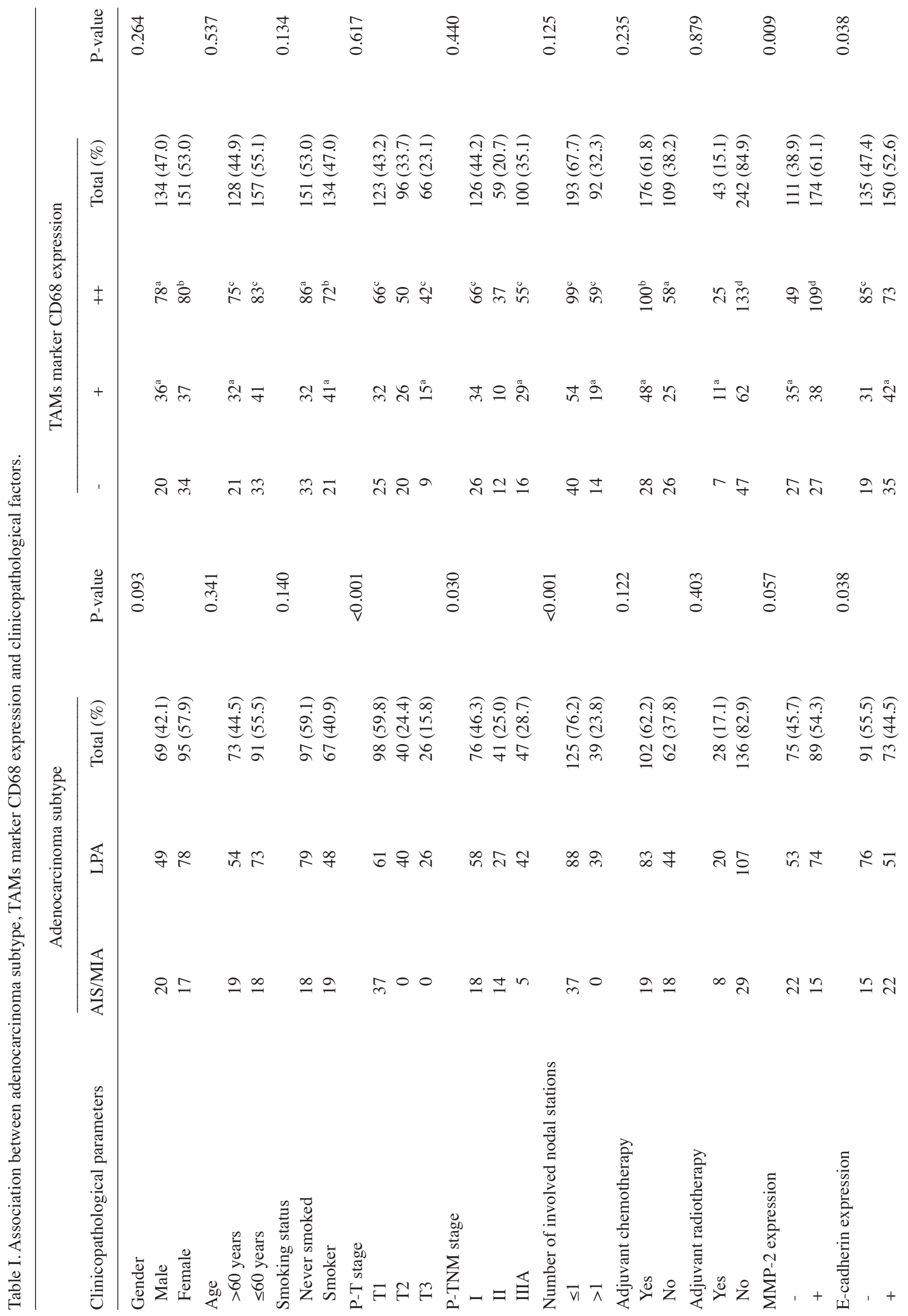


according to histological type, with 37 cases of AIS/MIA (13.0\%), 127 cases of LPA (44.6\%) and 121 cases of other cancer types $(42.4 \%)$. Five patients were excluded from the study, since their pathological types did not belong to AIS/MIA or LPA, but to other types of adenocarcinoma.

Immunohistochemistry staining results, and difference between AIS/MIA and LPA. CD68 was stained mostly in the cytoplasm of TAMs, but not in tumor cells, lymphocytes, plasmocytes or fibroblasts (Fig. 1A-D). Expression of IL-6 (Fig. 1E and F) and CSF-1 (Fig. 1G and H) was observed in the cytoplasm of tumor cells, with sporadic positive staining in inflammatory cells such as lymphocytes, macrophages and plasmocytes. E-cadherin positive staining was located in the cytomembrane (Fig. 1I and J), and the expression of Snail in the majority of tumor cells displayed a nuclear staining pattern (Fig. 1K). MMP-2 expression was also mainly observed in the cytoplasm of tumor cells (Fig. 1L).

Stepwise progression from AIS/MIA to invasive LPA was assumed. The clinicopathological differences between AIS/MIA and LPA were investigated. As shown in Table I, significant differences were present among postoperative (P)-T stage $(\mathrm{P}<0.001)$, $\mathrm{P}-\mathrm{TNM}$ stage $(\mathrm{P}=0.030)$, number of involved nodal stations $(\mathrm{P}<0.001)$, E-cadherin expression $(\mathrm{P}=0.038)$ and Snail expression $(\mathrm{P}=0.010)$. Gender, age, smoking status, reception of adjuvant chemotherapy or radiotherapy, and expression of MMP-2 were not significantly different between these two groups $(\mathrm{P}>0.05)$.

Infiltration of interstitialTAMs and their association with clinicopathological features. The median numbers of stromal TAMs $/ \mathrm{hpf}$ were 25.03 (mean \pm standard deviation, $24.73 \pm 16.87 \mathrm{TAMs} / \mathrm{hpf}$; range, $0.00-71.42 \mathrm{TAMs} / \mathrm{hpf})$. Based on the median numbers of TAMs, 54 patients (18.9\%) were CD68-, and 73 patients (25.6\%) were categorized into the low-TAMs group ( $\leq 25 \mathrm{TAMs} / \mathrm{hpf})$, while 158 patients $(55.5 \%)$ were categorized into the high-TAMs group (>25 TAMs/hpf). In order to investigate whether TAMs were associated with tumor progression, the association of CD68 expression levels and clinicopathological features was also illustrated in detail. The infiltration degree of TAMs in tumor stroma was higher in LPA than in AIS/MIA ( $\mathrm{P}=0.012)$. In addition, Snail and MMP-2 expression were positively correlated with the infiltration degree of TAMs $(\mathrm{P}=0.004$ and $\mathrm{P}=0.009$, respectively). By contrast, E-cadherin exhibited a significantly negative correlation with the infiltration degree of TAMs $(\mathrm{P}=0.038)$. In the CD68- group, the positive expression rate of E-cadherin was $35 / 54(64.8 \%)$. In the low-TAMs group, the positive expression rate of E-cadherin was 42/73 (57.5\%), while in the high-TAMs group, the positive expression rate of E-cadherin was 73/158 (46.2\%). Immunofluorescent double staining revealed that E-cadherin localized to the plasma membrane of cells in areas with low CD68+ TAMs density (Fig. 2A), while the expression of E-cadherin was compromised and partially lost in areas with high TAMs density (Fig. 2B).

Prognostic factors of lung adenocarcinoma. The 5-year OS and DFS rates were 44.0 and $40.0 \%$, respectively, for the total study population. Univariate analyses revealed that MMP-2, the EMT markers E-cadherin and Snail, P-T stage, P-TNM stage, number of involved nodal stations and reception of adjuvant radiotherapy 


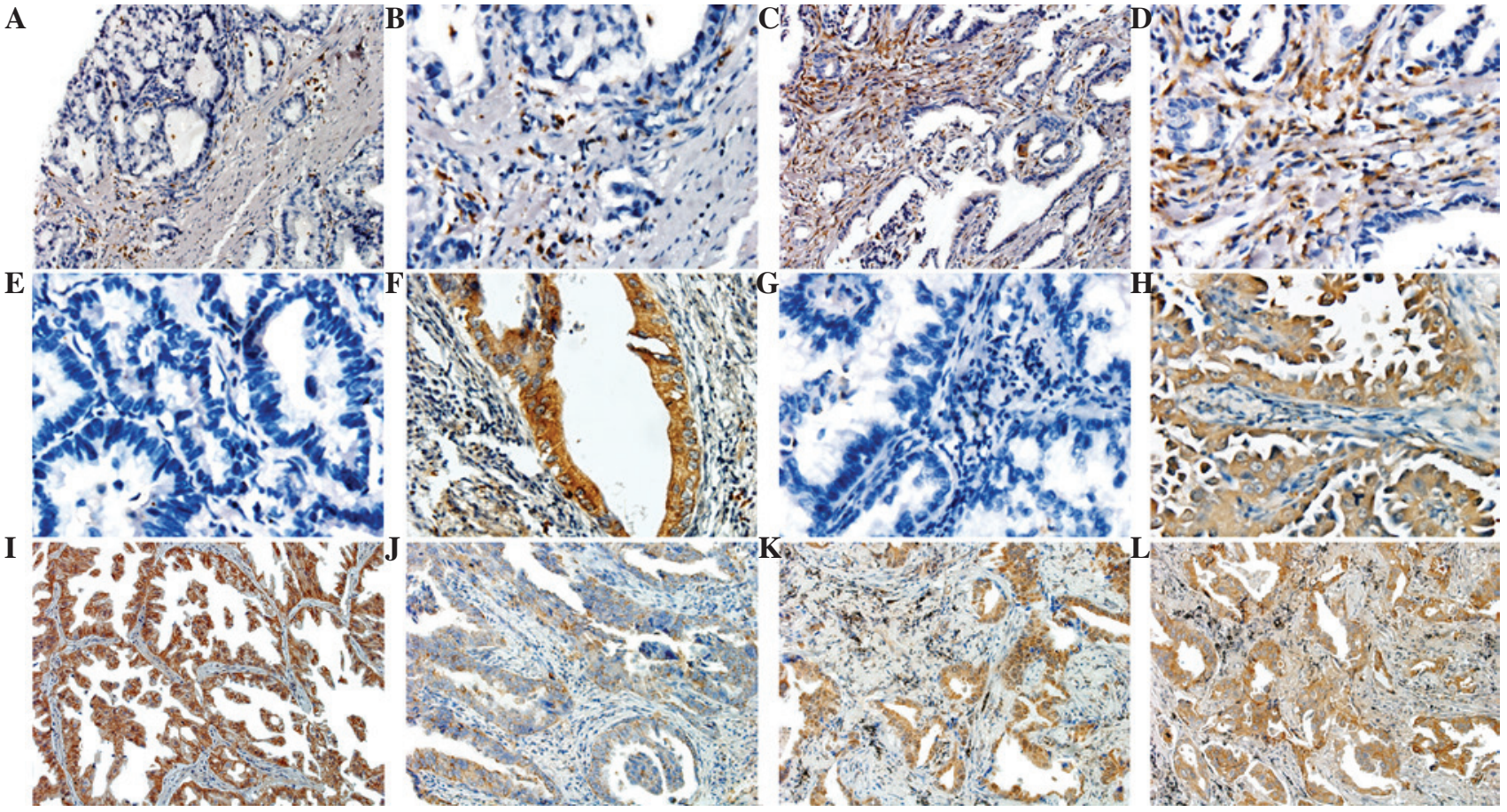

Figure 1. Typical expression images of immunochemistry staining in tissue microarrays. (A and B) Low expression of CD68 in MIA tissues. Original magnification, (A) x100 and (B) x200. (C and D) High expression of CD68 in LPA tissues. Original magnification, (C) x100 and (D) x200. (E) Negative expression of IL-6 in MIA tissues. Original magnification, x200. (F) Positive expression of IL-6 in LPA tissues. Original magnification, x200. (G) Negative expression of CSF-1 in MIA tissues. Original magnification, x200. (H) Positive expression of CSF-1 in LPA tissues. Original magnification, x200. (I) High E-cadherin expression in MIA tissues. Original magnification, x100. (J) Low E-cadherin expression in LPA tissues. Original magnification, x100. (K) High Snail expression in LPA tissues. Original magnification, x100. (L) High MMP-2 expression in LPA tissues. Original magnification, x100. CD, cluster of differentiation; MIA, minimally invasive adenocarcinoma; LPA, lepidic predominant adenocarcinoma; CSF, colony-stimulating factor; IL, interleukin; MMP, metalloproteinase.

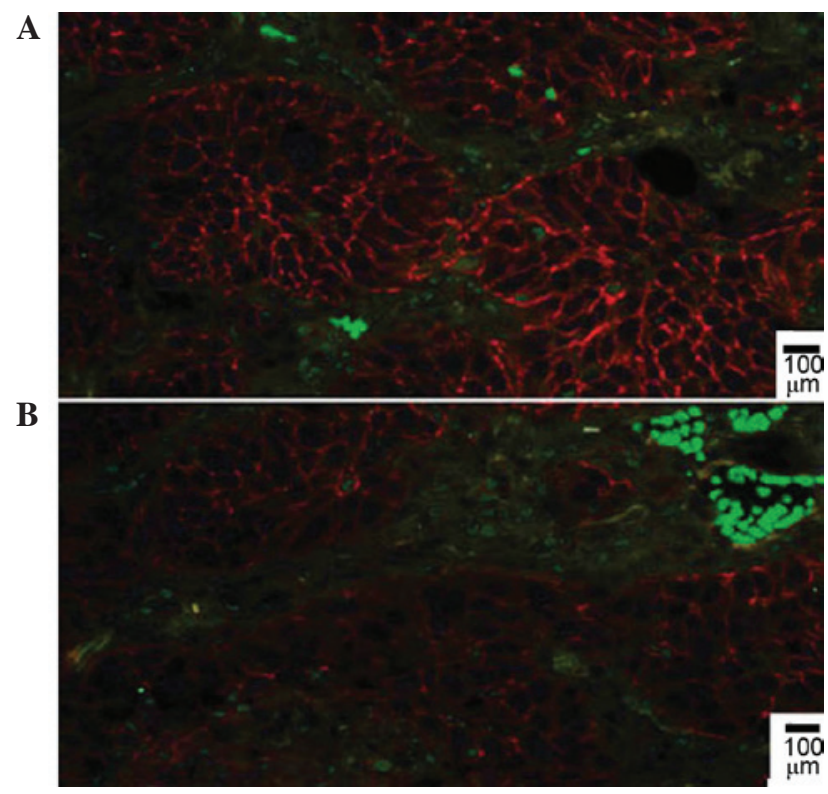

Figure 2. Double immunofluorescence staining for E-cadherin (red) and cluster of differentiation 68 (green) (A) in a macrophage-poor area and (B) in a macrophage-rich area. Original magnification, $\mathrm{x} 400$

or chemotherapy were significant prognostic factors $(\mathrm{P}<0.05$; Table II). Besides, a significant difference was noticed between AIS/MIA and LPA for both OS (65.7 vs. $38.6 \%, \mathrm{P}=0.008)$ and DFS (64.4 vs. $37.3 \%, \mathrm{P}=0.006)$. The survival data also revealed that the 5-year OS and DFS rates for the CD68+ group were worse than those for the CD68- group, but better than those for the CD68++ group (45.4 vs. 53.6 and $35.3 \%, \mathrm{P}=0.014$; and 40.3 vs. 51.1 and $33.4 \%, P=0.017$, respectively).

To elucidate the phenotype and biological function of TAMs, the levels of tumor-derived CSF-1 and IL-6 were analyzed, since these molecules are associated with TAMs functional plasticity $(10,11)$. CSF-1 and IL-6 could also be used as prognostic factors for survival ( $\mathrm{P}<0.001$, data not shown). Additional analysis demonstrated that the 5-year OS (19.9\%) and DFS (14.1\%) rates in the CD68+CSF-1+IL-6+ group were the worst, while the prognosis of the CD68+CSF-1-IL-6- group was the best (71.0 and $68.1 \%$, respectively). The 5 -year survival rates for the CD68- group and the CD68+CSF-1+IL-6- group or the CD68+CSF-1-IL-6+ group were intermediate between those of the group with the worst and the group with the best rates $(\mathrm{P}<0.001$; Fig. 3). Multivariate analysis determined that the combination of CD68/CSF-1/IL-6 [OS hazard ratio $(\mathrm{HR})=3.360$, DFS HR=4.179) remained significant and was an independent prognostic factor for survival $(\mathrm{P}<0.001$; Table III).

TAMs may serve an active role in facilitating AIS/MIA progression to $L P A$. In order to further validate that TAMs regulated the progression of lung adenocarcinoma, a stratified analysis was performed according to adenocarcinoma subtype and CD68 expression in TAMs (Table IV). Our results indicated that the 5-year OS and DFS rates in AIS/MIA patients with a number of TAMs $\leq 25$ were better than those in AIS/MIA patients with TAMs $>25(\mathrm{P}=0.019$ and $\mathrm{P}=0.014$, respectively; Fig. $4 \mathrm{~A}$ and $\mathrm{B})$. By contrast, in patients with 
Table II. Univariate survival analysis according to clinicopathological factors.

\begin{tabular}{|c|c|c|c|c|c|}
\hline Variables & Cases, n (\%) & 5YOSR, \% & P-value & 5YDFSR, \% & P-value \\
\hline Gender & & & 0.170 & & 0.194 \\
\hline Male & $134(47.0)$ & 39.1 & & 36.7 & \\
\hline Female & $151(53.0)$ & 45.3 & & 42.6 & \\
\hline Age & & & 0.943 & & 0.865 \\
\hline$>60$ years & $128(44.9)$ & 43.4 & & 41.2 & \\
\hline$\leq 60$ years & $157(55.1)$ & 41.4 & & 38.7 & \\
\hline Smoking status & & & 0.068 & & 0.087 \\
\hline Never smoked & $151(53.0)$ & 46.0 & & 43.4 & \\
\hline Smoker & $134(47.0)$ & 38.9 & & 36.0 & \\
\hline Adenocarcinoma subtype & & & 0.008 & & 0.006 \\
\hline AIS/MIA & 37 (22.6) & 65.7 & & 64.4 & \\
\hline LPA & $127(77.4)$ & 38.6 & & 37.3 & \\
\hline P-T stage & & & $<0.001$ & & $<0.001$ \\
\hline $\mathrm{T} 1$ & $123(43.2)$ & 59.8 & & 54.8 & \\
\hline $\mathrm{T} 2$ & $96(33.7)$ & 38.6 & & 36.4 & \\
\hline $\mathrm{T} 3$ & $66(23.1)$ & 17.4 & & 14.3 & \\
\hline P-TNM stage & & & $<0.001$ & & $<0.001$ \\
\hline $\mathrm{I}$ & $126(44.2)$ & 62.6 & & 58.1 & \\
\hline II & $59(20.7)$ & 38.3 & & 33.1 & \\
\hline IIIA & $100(35.1)$ & 19.5 & & 16.9 & \\
\hline Number of involved nodal stations & & & $<0.001$ & & $<0.001$ \\
\hline$\leq 1$ & $193(67.7)$ & 53.3 & & 49.1 & \\
\hline$>1$ & $92(32.3)$ & 19.2 & & 18.0 & \\
\hline Adjuvant chemotherapy & & & 0.025 & & 0.004 \\
\hline Yes & $176(61.8)$ & 39.3 & & 32.3 & \\
\hline No & $109(38.2)$ & 51.6 & & 50.2 & \\
\hline Adjuvant radiotherapy & & & 0.032 & & 0.009 \\
\hline Yes & $43(15.1)$ & 24.6 & & 15.5 & \\
\hline No & $242(84.9)$ & 45.6 & & 43.9 & \\
\hline CD68 expression & & & 0.014 & & 0.017 \\
\hline- & $54(18.9)$ & 53.6 & & 51.1 & \\
\hline+ & $73(25.6)$ & 45.4 & & 40.3 & \\
\hline++ & $158(55.5)$ & 35.3 & & 33.4 & \\
\hline CD68/CSF-1/IL-6 expression & & & $<0.001$ & & $<0.001$ \\
\hline CD68- & $54(18.9)$ & 45.4 & & 40.3 & \\
\hline CD68+CSF-1-IL-6- & $62(21.8)$ & 71.0 & & 68.1 & \\
\hline CD68+CSF-1+IL-6- & $39(13.7)$ & 47.6 & & 46.9 & \\
\hline CD68+CSF-1-IL-6+ & $33(11.6)$ & 44.7 & & 41.1 & \\
\hline CD68+CSF-1+IL-6+ & $97(34.0)$ & 19.9 & & 14.1 & \\
\hline MMP-2 expression & & & 0.010 & & 0.007 \\
\hline- & $111(38.9)$ & 52.5 & & 50.8 & \\
\hline+ & $174(61.1)$ & 36.3 & & 33.1 & \\
\hline E-cadherin expression & & & 0.038 & & 0.047 \\
\hline- & $135(47.4)$ & 35.7 & & 33.2 & \\
\hline+ & $150(52.6)$ & 50.5 & & 47.7 & \\
\hline Snail expression & & & 0.004 & & 0.002 \\
\hline- & $85(29.8)$ & 56.3 & & 51.4 & \\
\hline+ & $200(70.2)$ & 36.6 & & 33.9 & \\
\hline
\end{tabular}

P, postoperative TNM, tumor-node-metastasis stage; CD, cluster of differentiation; 5YOSR, 5-year overall survival rate; 5YDFSR, 5-year disease-free survival rate; AIS, adenocarcinoma in situ; MIA, minimally invasive adenocarcinoma; LPA, lepidic predominant adenocarcinoma; MMP, matrix metalloproteinase; CSF, colony-stimulating factor; IL, interleukin. 

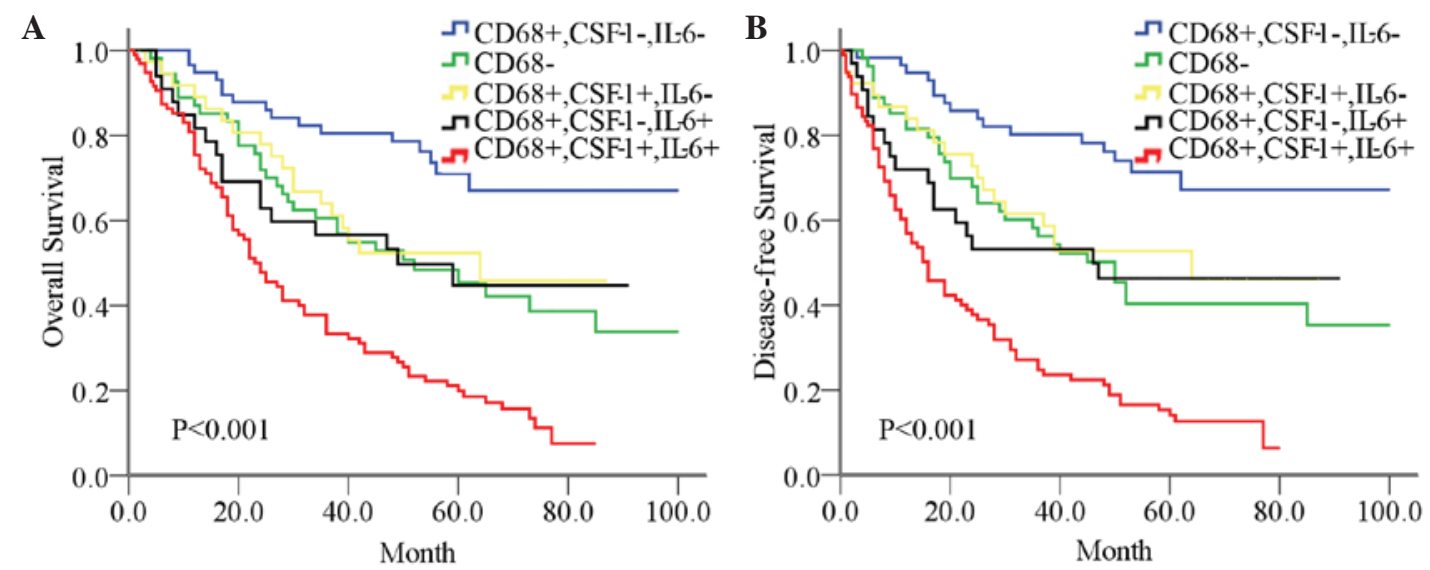

Figure 3. (A) Kaplan-Meier analysis of overall survival for the combination of CD68, CSF-1 and IL-6. (B) Kaplan-Meier analysis of disease-free survival for the combination of CD68, CSF-1 and IL-6. CSF-1, colony-stimulating factor-1; CD, cluster of differentiation; IL, interleukin.

A

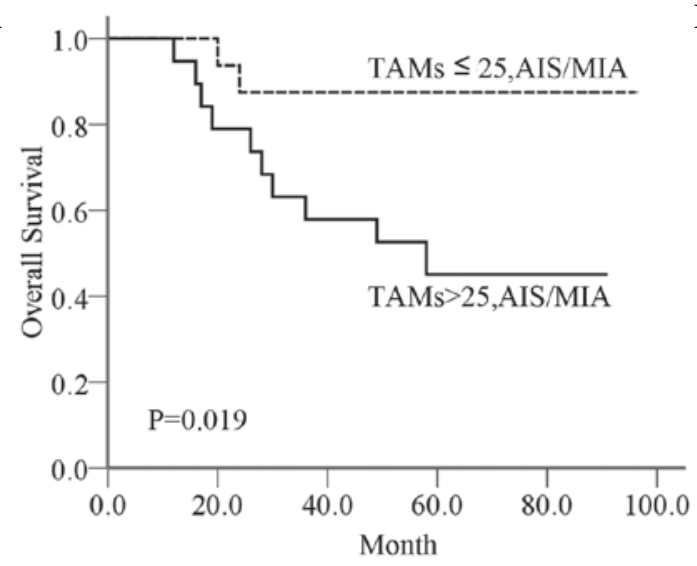

C

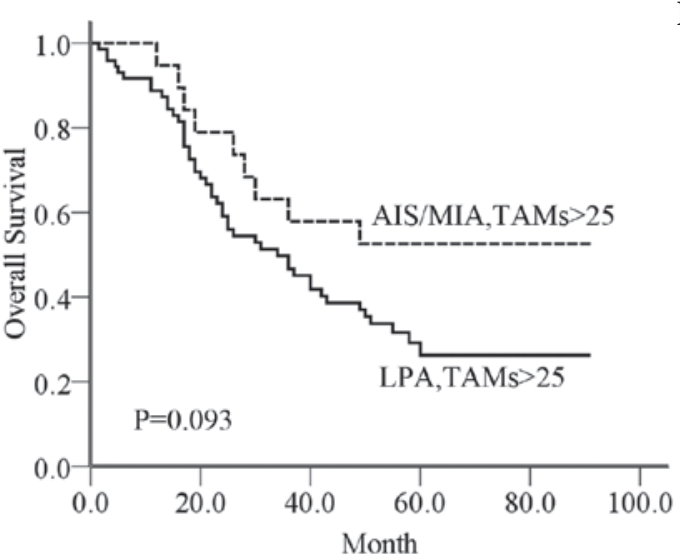

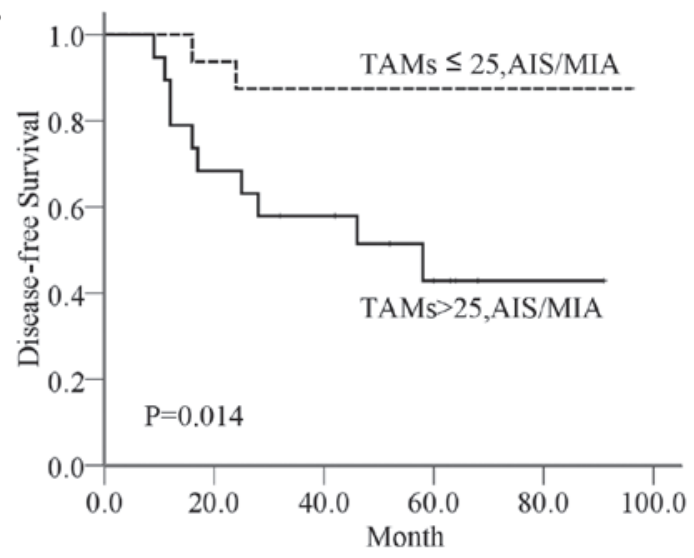

D

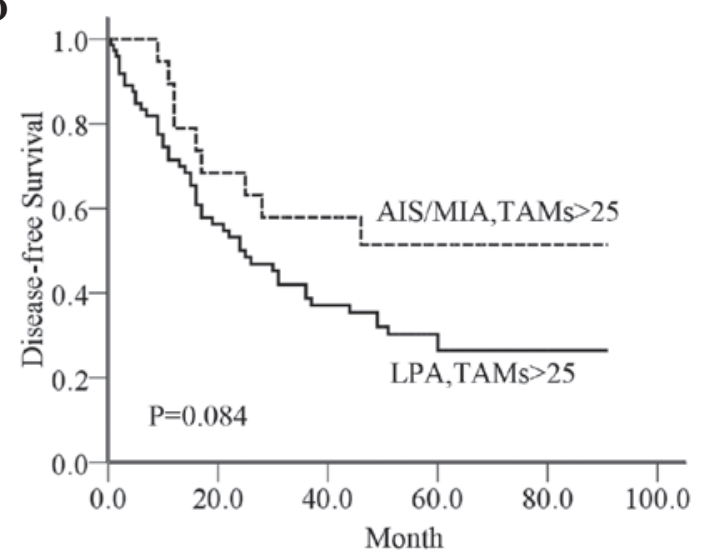

Figure 4. (A) Kaplan-Meier analysis of OS for the number of TAMs in AIS/MIA patients. (B) Kaplan-Meier analysis of DFS for the number of TAMs in AIS/MIA patients. (C) Comparison of OS in AIS/MIA patients with TAMs $>25$ and in LPA patients with TAMs $>25$. (D) Comparison of DFS in AIS/MIA patients with TAMs $>25$ and in LPA patients with TAMs >25. AIS, adenocarcinoma in situ; MIA, minimally invasive adenocarcinoma; TAMs, tumor-associated macrophages; LPA, lepidic predominant adenocarcinoma; OS, overall survival; DFS, disease-free survival.

TAMs $>25$, the 5-year OS and DFS rates were not significantly different between AIS/MIA and LPA $(\mathrm{P}=0.093$ and $\mathrm{P}=0.084$, respectively; Fig. 4C and D). In AIS/MIA patients, there was a similar statistically significant difference between the CD68+CSF-1+IL-6+ group and other groups (CD68-, CD68+CSF-1+IL-6-, CD68+CSF-1-IL-6+ and CD68+CSF-1-IL-6- groups) $(\mathrm{P}=0.008$ and $\mathrm{P}=0.012$, respectively; Fig. 5A and B). However, no significant difference in patients with positive expression for all three markers (CD68, CSF-1 and IL-6) was observed between AIS/MIA and LPA $(\mathrm{P}=0.580$ and $\mathrm{P}=0.301$, respectively; Fig. 5C and $\mathrm{D})$.

\section{Discussion}

Accumulating evidence has shown that TAMs in the tumor microenvironment can significantly enhance the malignant 
Table III. Multivariate analysis of factors associated with overall survival and disease-free survival.

\begin{tabular}{|c|c|c|c|c|}
\hline \multirow[b]{2}{*}{ Variables } & \multicolumn{2}{|c|}{ Overall survival } & \multicolumn{2}{|c|}{ Disease-free survival } \\
\hline & Hazard ratio $(95 \% \mathrm{CI})$ & P-value & Hazard ratio $(95 \% \mathrm{CI})$ & P-value \\
\hline $\begin{array}{l}\text { Adenocarcinoma subtype } \\
\text { (LPA vs. AIS/MIA) }\end{array}$ & $1.297(0.951-1.768)$ & 0.100 & $1.376(1.009-1.876)$ & 0.044 \\
\hline $\begin{array}{l}\text { Number of involved nodal } \\
\text { stations ( }>1 \text { vs. } \leq 1)\end{array}$ & $1.664(0.993-2.788)$ & 0.053 & $1.973(1.176-3.310)$ & 0.010 \\
\hline P-TNM stage (II and IIIA vs. I) & $2.021(1.119-3.649)$ & 0.020 & $1.862(1.028-3.371)$ & 0.040 \\
\hline P-T stage (T2 and T3 vs. T1) & $1.399(0.838-2.336)$ & 0.199 & $1.461(0.893-2.390)$ & 0.131 \\
\hline Adjuvant chemotherapy (yes vs. no) & $0.625(0.351-1.112)$ & 0.110 & $0.865(0.493-1.517)$ & 0.613 \\
\hline Adjuvant radiotherapy (yes vs. no) & $1.430(0.864-2.366)$ & 0.164 & $1.759(1.062-2.915)$ & 0.028 \\
\hline E-cadherin (+ vs. - ) & $0.511(0.323-0.810)$ & 0.004 & $0.587(0.375-0.919)$ & 0.020 \\
\hline Snail (+ vs. -) & $0.995(0.546-1.815)$ & 0.987 & $1.143(0.627-2.083)$ & 0.662 \\
\hline MMP-2 (+ vs. -) & $1.414(0.856-2.333)$ & 0.176 & $1.362(0.815-2.274)$ & 0.238 \\
\hline CD68+CSF-1+IL-6+ vs. others ${ }^{\mathrm{a}}$ & $3.360(2.093-5.396)$ & $<0.001$ & $4.179(2.628-6.646)$ & $<0.001$ \\
\hline
\end{tabular}

aOthers include CD68+CSF-1+IL-6-, CD68+CSF-1-IL-6+, CD68+CSF-1-IL-6- and CD68-. P, postoperative; TNM, tumor-node-metastasis; CI, confidence interval; AIS, adenocarcinoma in situ; MIA, minimally invasive adenocarcinoma; LPA, lepidic predominant adenocarcinoma; CSF, colony-stimulating factor; IL, interleukin; MMP, matrix metalloproteinase; CD, cluster of differentiation.

Table IV. Stratified analysis according to adenocarcinoma subtype and number of CD68+ TAMs.

\begin{tabular}{|c|c|c|c|c|c|}
\hline Variables & Cases, $\%$ & $5 Y O S R, \%$ & P-value & 5YDFSR, \% & P-value \\
\hline AIS/MIA, number of TAMs & & & 0.019 & & 0.014 \\
\hline AIS/MIA, TAMs > 25 & 14 & 45.1 & & 42.9 & \\
\hline AIS/MIA, TAMs $\leq 25$ & 23 & 87.5 & & 87.5 & \\
\hline TAMs $>25$, adenocarcinoma subtype & & & 0.093 & & 0.084 \\
\hline TAMs $>25$, AIS/MIA & 14 & 45.1 & & 42.9 & \\
\hline TAMs $>25$, LPA & 81 & 26.1 & & 25.9 & \\
\hline AIS/MIA, CD68/CSF-1/IL-6 & & & 0.008 & & 0.012 \\
\hline AIS/MIA, CD68+CSF-1+IL-6+ & 6 & 20.0 & & 20.0 & \\
\hline AIS/MIA, others ${ }^{\mathrm{a}}$ & 31 & 73.0 & & 73.0 & \\
\hline CD68+CSF-1+IL-6+, adenocarcinoma subtype & & & 0.580 & & 0.301 \\
\hline CD68+CSF-1+IL-6+, AIS/MIA & 6 & 20.0 & & 20.0 & \\
\hline CD68+CSF-1+IL-6+, LPA & 55 & 13.1 & & 8.5 & \\
\hline
\end{tabular}

aOthers include CD68+CSF-1+IL-6-, CD68+CSF-1-IL-6+, CD68+CSF-1-IL-6- and CD68-. 5YOSR, 5-year overall survival rate; 5YDFSR, 5-year disease-free survival rate; TAMs, tumor-associated macrophages; CSF, colony-stimulating factor; IL, interleukin; MMP, matrix metalloproteinase; CD, cluster of differentiation; AIS, adenocarcinoma in situ; MIA, minimally invasive adenocarcinoma; LPA, lepidic predominant adenocarcinoma.

phenotypes of tumors by promoting tumor growth and invasiveness (10,11). Experimental models have demonstrated that the lack of macrophage recruitment to the tumor site results in decreased tumorigenic ability $(12,13)$, and clinical evidence has shown a strongly correlation between increased TAMs density in tumor stroma and poor prognosis in different types of solid tumors (14-16). Along with these previous results, our study demonstrated that high infiltration of TAMs was negatively associated with human lung adenocarcinoma prognosis $(\mathrm{P}<0.05)$, and that the infiltration degree of TAMs in the tumor stroma was higher in LPA than in AIS/MIA $(\mathrm{P}<0.05)$. These results study indicated that highly infiltrating TAMs may promote tumor invasion and progression.

Furthermore, in the tumor microenvironment, neoplastic cells can shape the differentiation and functional orientation of TAMs, which, in turn, exert several pro-tumoral functions, including secretion of growth factors and matrix proteases, promotion of angiogenesis, and suppression of adaptive immunity (17-19). Recent studies have also shown that TAMs are recruited into tumor regions by a range of bioactive chemokines 
A

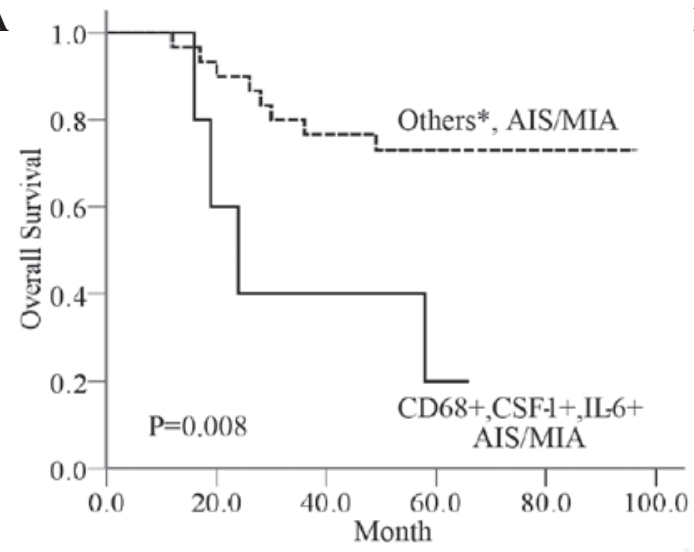

C

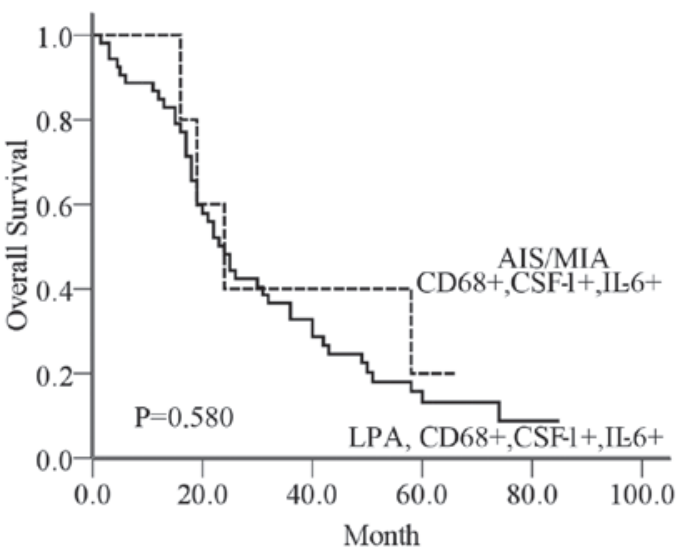

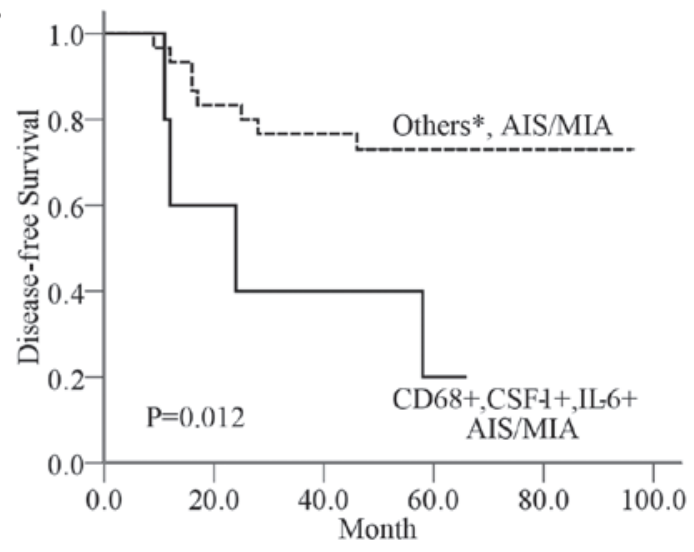

D

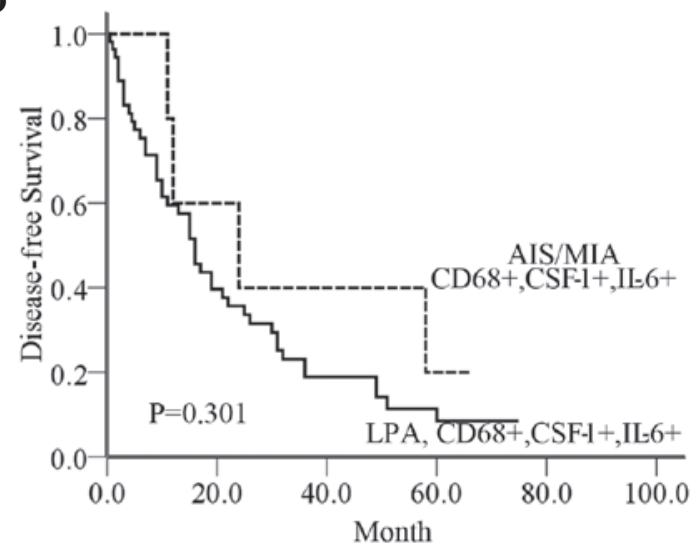

Figure 5. (A) OS in patients with AIS/MIA in the CD68+CSF-1+IL-6+ group compared with other groups, including the CD68-, CD68+CSF-1+IL-6-, CD68+CSF-1-IL-6+ and CD68+CSF-1-IL-6- groups. (B) DFS in patients with AIS/MIA in the CD68+ CSF-1+ IL-6+ group compared with other groups (C) Comparison of OS in AIS/MIA patients with CD68+CSF-1+IL-6+ and in LPA patients with CD68+CSF-1+IL-6+. (D) Comparison of DFS in AIS/MIA patients with CD68+CSF-1+IL-6+ and in LPA patients with CD68+CSF-1+IL-6+. *Others include the CD68+CSF-1+IL-6- group, the CD68+CSF-1-IL-6+ group, the CD68+CSF-1-IL-6- group and the CD68- group. AIS, adenocarcinoma in situ; MIA, minimally invasive adenocarcinoma; CD, cluster of differentiation; CSF, colony-stimulating factor; IL, interleukin; OS, overall survival; DFS, disease-free survival.

in the tumor microenvironment, including CSF-1, IL-6, IL-10, which are mainly produced by the tumor cells themselves, and are educated toward a tumor-promoting phenotype (20-22). The study these cytokines is currently ongoing at our group, and the interaction between cancer cells and TAMs is under way. The detailed signaling pathway and underlying regulatory mechanisms still require to be further in-depth investigated. In the present study, univariate and multivariate analyses for survival demonstrated that the combination of CD68, CSF-1 and IL-6 was the most significant and independent prognostic factor $(\mathrm{P}<0.001)$. The 5-year survival rates in the group with positive expression of CD68, CSF-1 and IL- 6 were the worst. The prognosis of the CD68+CSF-1-IL-6- group was the best, and better than that of the CD68- group. These findings have been confirmed by previous studies. For instance, Duluc et al (23) noticed that tumor-associated leukemia inhibitory factor and IL-6, which are present at high concentrations in ovarian cancer ascites, redirect monocyte differentiation into tumor-promoting TAMs by increasing CSF-1 consumption. In addition, Dijkgraaf et al (24) considered that prostaglandin E2 and IL-6 were associated with chemoresistance and tumor-induced differentiation of tumor-promoting macrophages. Based on these results, it was assumed that the combination of CSF-1 and IL-6 had the ability to strongly induce the formation of tumor-promoting TAMs.
In order to further validate that TAMs regulated the progression of lung adenocarcinoma, a stratified analysis was performed according to AIS/MIA type and CD68 expression in TAMs. In our study, AIS/MIA patients had a significantly better prognosis than LPA patients, whereas LPA had significantly worse prognosis $(\mathrm{P}<0.05)$. The 5 -year survival rates in AIS/MIA patients with TAMs $\leq 25$ were better than those in AIS/MIA patients with TAMs $>25(\mathrm{P}<0.05)$. In AIS/MIA patients, there was a similar statistically significant difference between the CD68+CSF-1+IL-6+ group and the other groups (CD68-, CD68+CSF-1+IL-6-, CD68+CSF-1-IL-6+ and CD68+CSF-1-IL-6- groups) $(\mathrm{P}<0.05)$. By contrast, in patients with TAMs $>25$ and in patients with positive expression of CD68, CSF-1 and IL-6, the survival rates were not significantly different between AIS/MIA and LPA ( $\mathrm{P}>0.05)$. Accordingly, it was likely that TAMs may play an active role in facilitating AIS/MIA progression to LPA.

A characterized progression that epithelial-derived tumor cells undergo is termed EMT, which involves loss of polarity and adhesion, increased mobility and invasiveness, and acquisition of an invasive mesenchymal phenotype $(6,25,26)$. The loss of E-cadherin has been shown to be associated with increased tumor invasiveness, metastasis and poor prognosis $(25,27)$. Snail and Slug have been established as repressors of E-cadherin, one of the key molecules in the EMT 
process, both in early development and in cancer progression (27). MMP-2 is regarded as a crucial enzyme for tumor progression, invasion and metastasis due to its capability to degrade basement membrane components (28). Therefore, we hypothesized that TAMs may facilitate adenocarcinoma progression by inducing EMT and degrading the extracellular matrix, thereby contributing to tumor heterogeneity and grade. The results of the present study indicated that Snail and MMP-2 expression were positively correlated with the infiltration degree of TAMs, whereas E-cadherin expression exhibited a modest negative correlation with CD68+ densities $(\mathrm{P}<0.05)$. It was certified that various cytokines secreted by activated TAMs can induce the EMT phenotype in cancer cells $(29,30)$. As EMT is associated with both drug resistance and patient relapse, it is possible to speculate that therapeutic targeting of TAMs could improve disease outcome. Considering the importance of TAMs in promoting tumor progression, treatment only targeting the malignant cells would not be efficient. The results presented herein also provide important new insight into the significance of cancer-stroma cell interactions in influencing the outcome of cancer therapy, which should be helpful for the rational design of an anticancer strategy.

\section{Acknowledgements}

The present study was supported by the National Natural Science Foundation of China (Beijing, China; grant nos. 81201649 and 30801377) and Tianjin Natural Science Foundation of China (Tianjin, China; grant no. 11JCYBJC13300).

\section{References}

1. Devesa SS, Bray F, Vizcaino AP and Parkin DM: International lung cancer trends by histologic type: Male: Female differences diminishing and adenocarcinoma rates rising. Int $\mathrm{J}$ Cancer 117: 294-299, 2005.

2. Soh J, Toyooka S, Ichihara S, Asano H, Kobayashi N, Suehisa H, Otani H, Yamamoto H, Ichimura K, Kiura K, et al: Sequential molecular changes during multistage pathogenesis of smal peripheral adenocarcinomas of the lung. J Thorac Oncol 3: 340-347, 2008

3. Mantovani A, Allavena P, Sica A and Balkwill F: Cancer-related inflammation. Nature 454: 436-444, 2008.

4. Solinas G, Germano G, Mantovani A and Allavena P: Tumor-associated macrophages (TAM) as major players of the cancer-related inflammation. J Leukoc Biol 86: 1065-1073, 2009.

5. Mantovani A, Schioppa T, Porta C, Allavena P and Sica A: Role of tumor-associated macrophages in tumor progression and invasion. Cancer Metastasis Rev 25: 315-322, 2006.

6. Thiery JP, Acloque H, Huang RY and Nieto MA: Epithelial-mesenchymal transitions in development and disease. Cell 139: 871-890, 2009.

7. Detterbeck FC, Boffa DJ and Tanoue LT: The new lung cancer staging system. Chest 136: 260-271, 2009.

8. Richardsen E, Uglehus RD, Due J, Busch C and Busund LT: The prognostic impact of CSF-1, CSF-1 receptor, CD68 and CD3 in prostatic carcinoma. Histopathology 53: 30-38, 2008.

9. Gwak JM, Jang MH, Kim DI, Seo AN and Park SY: Prognostic value of tumor-associated macrophages according to histologic locations and hormone receptor status in breast cancer. PLoS One 10: e0125728, 2015.

10. Quail DF and Joyce JA: Microenvironmental regulation of tumor progression and metastasis. Nat Med 19: 1423-1437, 2013.
11. Hu M and Polyak K: Microenvironmental regulation of cancer development. Curr Opin Genet Dev 18: 27-34, 2008.

12. Zhang W, Zhu XD, Sun HC, Xiong YQ, Zhuang PY, Xu HX, Kong LQ, Wang L, Wu WZ and Tang ZY: Depletion of tumor-associated macrophages enhances the effect of sorafenib in metastatic liver cancer models by antimetastatic and antiangiogenic effects. Clin Cancer Res 16: 3420-3430, 2010.

13. Zabuawala T, Taffany DA, Sharma SM, Merchant A, Adair B, Srinivasan R, Rosol TJ, Fernandez S, Huang K, Leone G and Ostrowski MC: An ets2-driven transcriptional program in tumor-associated macrophages promotes tumor metastasis. Cancer Res 70: 1323-1333, 2010.

14. Subimerb C, Pinlaor S, Khuntikeo N, Leelayuwat C, Morris A, McGrath MS and Wongkham S: Tissue invasive macrophage density is correlated with prognosis in cholangiocarcinoma. Mol Med Rep 3: 597-605, 2010.

15. Zhang QW, Liu L, Gong CY, Shi HS, Zeng YH, Wang XZ, Zhao YW and Wei YQ: Prognostic significance of tumor-associated macrophages in solid tumor: A meta-analysis of the literature. PloS One 7: e50946, 2012.

16. Steidl C, Lee T, Shah SP, Farinha P, Han G, Nayar T, Delaney A, Jones SJ, Iqbal J, Weisenburger DD, et al: Tumor-associated macrophages and survival in classic Hodgkin's lymphoma. N Engl J Med 362: 875-885, 2010.

17. Qian BZ and Pollard JW: Macrophage diversity enhances tumor progression and metastasis. Cell 141: 39-51, 2010.

18. Sica A, Larghi P, Mancino A, Rubino L, Porta C, Totaro MG, Rimoldi M, Biswas SK, Allavena P and Mantovani A: Macrophage polarization in tumour progression. Semin Cancer Biol 18: 349-355, 2008.

19. Sica A and Bronte V: Altered macrophage differentiation and immune dysfunction in tumor development. J Clin Invest 117: 1155-1166, 2007

20. Lippitz BE: Cytokine patterns in patients with cancer: A systematic review. Lancet Oncol 14: e218-e228, 2013.

21. Bonecchi R, Locati $M$ and Mantovani A: Chemokines and cancer: A fatal attraction. Cancer cell 19: 434-435, 2011.

22. Sica A, Allavena P and Mantovani A: Cancer related inflammation: The macrophage connection. Cancer Lett 267: 204-215, 2008.

23. Duluc D, Delneste Y, Tan F, Moles MP, Grimaud L, Lenoir J, Preisser L, Anegon I, Catala L, Ifrah N, et al: Tumor-associated leukemia inhibitory factor and IL-6 skew monocyte differentiation into tumor-associated macrophage-like cells. Blood 110: 4319-4330, 2007.

24. Dijkgraaf EM, Heusinkveld M, Tummers B, Vogelpoel LT, Goedemans R, Jha V, Nortier JW, Welters MJ, Kroep JR and van der Burg SH: Chemotherapy alters monocyte differentiation to favor generation of cancer-supporting M2 macrophages in the tumor micro-environment. Cancer Res 73: 2480-2492, 2013.

25. Hugo H, Ackland ML, Blick T, Lawrence MG, Clements JA, Williams ED and Thompson EW: Epithelial-mesenchymal and mesenchymal-epithelial transitions in carcinoma progression. J Cell Physiol 213: 374-383, 2007.

26. Yilmaz M and Christofori G: EMT, the cytoskeleton, and cancer cell invasion. Cancer Metastasis Rev 28: 15-33, 2009.

27. Yang $\mathbf{J}$ and Weinberg RA: Epithelial-mesenchymal transition: At the crossroads of development and tumor metastasis. Dev Cell 14: 818-829, 2008.

28. González-Arriaga P, Pascual T, García-Alvarez A Fernández-Somoano A, López-Cima MF and Tardón A: Genetic polymorphisms in MMP-2, 9 and 3 genes modify lung cancer risk and survival. BMC Cancer 12: 121, 2012.

29. Bonde AK, Tischler V, Kumar S, Soltermann A and Schwendener RA: Intratumoral macrophages contribute to epithelial-mesenchymal transition in solid tumors. BMC Cancer 12: 35, 2012.

30. Gao D, Vahdat LT, Wong S, Chang JC and Mittal V: Microenvironmental regulation of epithelial-mesenchymal transitions in cancer. Cancer Res 72: 4883-4889, 2012. 